\title{
Investigating transcriptional regulation of Plasmodium falciparum upon drug perturbation
}

\author{
Tharina van Brummlen*, John WW Becker, Dalu T Mancama, Heinrich Hoppe \\ From Parasite to Prevention: Advances in the understanding of malaria \\ Edinburgh, UK. 20-22 October 2010
}

Gene regulation of the malaria parasite Plasmodium falciparum has proven to be complex with evidence supporting both transcriptional $[1,2]$ and post-transcriptional level control $[3,4]$. Transcriptional profiling of environmentally perturbed malaria parasites can reveal functionally related genes with common regulatory mechanisms that are responsive to external stimuli [2]. By interrogating microarray data derived from P. falciparum populations treated with several drug classes, a subset of four genes potentially involved with the parasite's survival mechanisms following drug perturbation were identified, i.e. general stress response genes. In addition, data from two independent functional genomics investigations on the response of $P$. falciparum parasites to polyamine biosynthesis inhibitors (DFMO/MDL73811 co-inhibition [5] and cyclohexylamine [6]), revealed four genes of which the transcript abundance appear to be uniquely affected upon polyamine depletion only $[5,6]$, i.e. perturbation-specific stress response genes. The regulation of this generalized versus perturbation-specific transcriptional responses was subsequently investigated.

In order to investigate transcriptional regulation of $P$. falciparum, stable transfection methodology was established through the use of green fluorescent protein and luciferase as transgene expression markers [7]. Subsequently, the regions $1500 \mathrm{bp}$ upstream of the coding sequence (presumably containing the promoters) of the eight genes were inserted into the transfection vector driving luciferase expression, with the heat shock protein 86 promoter as control. Parasite transfection was performed and transfectants were selected for resistance against the antifolate WR99210.

Stable lines of the $P$. falciparum transfectants were exposed to a variety of compounds from different antimalarial drug classes including two polyamine biosynthesis

CSIR, Biosciences, PO Box 395, Pretoria, 0001, South Africa inhibitors (i.e. artemisinin, mefloquine,doxycycline, DFMO and cyclohexylamine) and luciferase activity was quantified as a measure of promoter activity. The altered luciferase activity was used to determine whether the observed transcriptional response upon drug perturbation were indeed the result of true transcriptional control at the promoter level as opposed to alteration of mRNA stability or other post-transcriptional means.

Published: 20 October 2010

\section{References}

1. De Silva E, Gehrke A, Olszewski K, León I, Chahal J, Bulyk M, Llinás M: Specific DNA-binding by Apicomplexan AP2 transcription factors. Proc Natl Acad Sci USA 2008, 105:8393-8.

2. Hu G, Cabrera A, Kono M, Mok S, Chaal B, Haase S, Engelberg K, Cheemadan S, Spielmann T, Preiser $P$, et al: Transcriptional profiling of growth perturbations of the human malaria parasite Plasmodium falciparum. Nat Biotechnol 2010, 28:91-8.

3. Mair G, Braks J, Garver L, Dimopoulos G, Hall N, Wiegant J, Dirks R, Khan S, Janse C, Waters A: Translational repression is essential for Plasmodium sexual development and mediated by DDX6-type RNA helicase. Science 2006, 313:667-9.

4. Shock J, Fischer K, DeRisi J: Whole-genome analysis of mRNA decay in Plasmodium falciparum reveals a global lengthening of mRNA half-life during the intra-erythrocytic development cycle. Genome Biol 2007, 8:R134.

5. van Brummelen AC, Olszewski KL, Wilinski D, Llinas M, Louw Al, Birkholtz L$\mathrm{M}$ : Co-inhibition of Plasmodium falciparum S-adenosylmethionine decarboxylase/ornithine decarboxylase reveals perturbation-specific compensatory mechanisms by transcriptome, proteome, and metabolome analyses. J Biol Chem 2009, 284:4635-46.

6. Becker J, Mtwisha L, Crampton B, Stoychev S, Van Brummelen A, Reeksting S, Louw A, Birkholtz L-M, Mancama D: Plasmodium falciparum spermidine synthase inhibition results in unique perturbation-specific effects observed on transcript, protein and metabolite levels. BMC Genomics 2010, 11:235.

7. Van Brummelen AC, Becker JWW, et al: Establishing malaria parasite transfection technology in South Africa. In 22nd South African Society for Biochemistry and Molecular Biology Congress 2010, South Africa: Bloemfontein, South Africa.

doi:10.1186/1475-2875-9-S2-032

Cite this article as: van Brummlen et al:: Investigating transcriptional regulation of Plasmodium falciparum upon drug perturbation. Malaria Journal 2010 9(Suppl 2):O32. 